\title{
CSF Alzheimer's disease-like pattern in corticobasal syndrome: evidence for a distinct disorder
}

\author{
Barbara Borroni, ${ }^{1}$ Enrico Premi, ${ }^{1}$ Chiara Agosti, ${ }^{1}$ Antonella Alberici, ${ }^{1}$ Carlo Cerini, ${ }^{1}$ \\ Silvana Archetti, ${ }^{2}$ Alessia Lanari, ${ }^{3}$ Barbara Paghera, ${ }^{4}$ Silvia Lucchini, ${ }^{4}$ Luigi Caimi, ${ }^{2}$ \\ Alessandro Padovani ${ }^{1}$
}

${ }^{1}$ Centre for Ageing Brain and Neurodegenerative Disorders, Neurology Unit, University of Brescia, Brescia, Italy

${ }^{2}$ Laboratories of Biotechnology, Brescia Hospital, Brescia, Italy ${ }^{3}$ Neurology Unit, Mantova Hospital, Mantova, Italy ${ }^{4}$ Nuclear Medicine Unit, University of Brescia, Brescia, Italy

\section{Correspondence to} Dr B Borroni, Clinica Neurologica, Università degli Studi di Brescia, Piazza Spedali Civili 1, Brescia, Italy; bborroni@inwind.it

Received 21 June 2010 Revised 20 September 2010 Accepted 24 December 2010 Published Online First 22 February 2011
Background Corticobasal syndrome (CBS) has a heterogeneous neuropathological spectrum, ranging from the classical corticobasal degeneration to Alzheimer's disease (AD). The neuropathology of CBS is still unpredictable. CSF tau/abeta ratio is a reliable marker of $A D$.

Objective To evaluate the presence of a distinct clinical and neuroimaging CBS phenotype according to CSF pattern.

Methods 30 patients fulfilling current clinical criteria for CBS entered the study. Each patient underwent a clinical and standardised neuropsychological assessment, and CSF analysis (total tau and abeta42 dosages). CSF AD-like pattern and CSF non-AD like pattern (nAD-like) were identified. In 23 CBS cases, ${ }^{99 m}$ Tc-ECD single photon emission computed tomography (SPECT) scan was performed and analysed by statistical parametric mapping. Results CSF AD-like pattern was reported in six cases (20\%). The two subgroups did not differ in demographic characteristics or global cognitive impairment. The AD-like group showed greater impairment of memory performances, language and psychomotor speed while the nAD-like group had more severe extrapyramidal syndrome with comparable apraxia scores. Voxel by voxel analysis on SPECT images demonstrated that CBS AD-like patients had greater hypoperfusion in the brain areas typically affected by $A D$ - namely, precuneus, posterior cingulate and hippocampus, bilaterally — compared with nAD-like patients $(p<0.001)$. No clusters above the pre-established threshold were detected when nAD-like were compared with AD-like patients.

Conclusions CSF AD-like profile in CBS is associated with earlier memory impairment and brain abnormalities typically found in classical $A D$. These findings argue for the usefulness of CSF testing to identify $A D$ in CBS, and might suggest a different pharmacological approach on the basis of biological data.

\section{INTRODUCTION}

Current clinical criteria for corticobasal syndrome (CBS) describe this disorder as an asymmetric, akinetic rigid syndrome, levodopa resistant, associated with prominent apraxia, cortical sensory loss, focal reflex myoclonus, dystonia, alien limb phenomena and without early dementia. ${ }^{1-3}$

As originally conceived, the neuropathology of the disease was considered as a specific disorder, namely corticobasal degeneration (CBD), characterised by cortical degeneration with swollen 'achromatic' neurons, neuronal loss in the substantia nigra and extensive neuronal and glial cytoplasmic tau positive inclusions. ${ }^{1}$ However, more recently, there is growing evidence that patients with clinically classic CBS have alternative pathologies. ${ }^{4}$ It has been reported that CBS is frequently associated with Alzheimer's disease (AD) pathology which is present in $15-20 \%$ of cases. ${ }^{5-8}$ Notwithstanding, the neuropathological features of CBS are still unpredictable in life.

Two retrospective studies tried to identify predictors in vivo of the specific neuropathology by comparing the clinical features of patients presenting with CBS who had either pathological changes of $\mathrm{CBD}$ or $\mathrm{AD}$ at post mortem. The first study suggested that early episodic memory impairment versus early behavioural symptomatology appears to best differentiate $\mathrm{AD}$ from $\mathrm{CBD},{ }^{9}$ while the other did not report any neuropsychological difference in the different neuropathological subtypes. ${ }^{10}$

Detecting $\mathrm{AD}$ in $\mathrm{CBS}$ has several clinical implications and might be associated with a different disease course and specific therapeutic approaches.

It has been widely demonstrated that $\mathrm{AD}$ is characterised by a significant decrease in the CSF tau/abeta42 ratio, which allows $\mathrm{AD}$ to be distinguished from other neurodegenerative disorders. ${ }^{11} 12$ The high sensitivity and specificity of the biomarker strongly suggests the introduction of the CSF tau/abeta42 ratio into current clinical criteria to further support the diagnosis, even at the preclinical disease stages. ${ }^{13}$ As the biological marker mirrors the pathogenetic mechanisms of $\mathrm{AD}$, it can be hypothesised that the CSF tau/abeta42 ratio might identify $\mathrm{AD}$ not only in the presymptomatic phase but also in the atypical presentation of the disorder.

These observations defined the aims of the present work. We sought to: (1) evaluate the presence and percentage of CSF AD-like pattern-that is, high CSF tau/abeta ratio-in a consecutive sample of CBS patients who underwent lumbar puncture; (2) assess the neuropsychological profile in $\mathrm{AD}$-like $\mathrm{CBS}$ patients, thus fulfilling current clinical criteria of CBS but with CSF AD-like abnormalities, and in non-AD like (nAD-like) CBS; and (3) evaluate the cerebral blood perfusion correlates at neuroimaging in the two groups.

\section{METHODS Subjects}

Thirty CBS patients (21 men and nine women; mean age $\pm S D 63.5 \pm 8.9$ years) recruited from the 
Centre for Neurodegenerative Diseases and the Centre for Movement Disorders, University of Brescia, Italy, entered the study.

All subjects underwent a somatic and neurological evaluation, and routine laboratory examination, and received a brain structural study.

The following essential diagnostic core criteria were fulfilled by all subjects: (a) insidious onset and gradual progression, (b) asymmetrical presentation, (c) akinetic rigid syndrome, (d) apraxia, as measured by de Renzi apraxia test, ${ }^{14}$ and (e) lack of sustained response to levodopa therapy. The supportive criteria included: (a) myoclonus, (b) limb dystonia, (c) cortical sensory loss and (d) alien limb phenomena. In each patient, the extrapyramidal syndrome developed first and was followed by cognitive changes.

Moreover, all CBS patients had been followed for at least 1 year after entering the study and the diagnosis of CBS was confirmed in all cases at the follow-up evaluation by clinical and neurological re-examination.

Stringent exclusion criteria were applied, as follows: (a) early dementia, early vertical gaze palsy, rest tremor, severe autonomic disturbances, sustained responsiveness to levodopa; (b) cerebrovascular disorders, hydrocephalus and intracranial mass, documented by MRI; (c) a history of traumatic brain injury or another neurological disease; and (d) significant medical problems.

All participants were right handed and were made fully aware of the aims of the research. Informed consent was sought from all subjects. The study was conducted in accordance with local clinical research regulations and conformed to the Helsinki Declaration.

\section{Clinical and neuropsychological assessment}

Motor impairment was evaluated by the motor section of the Unified Parkinson's Disease Rating Scale (UPDRS-III). ${ }^{15}$ Instrumental Activities of Daily Living and Basic Activities of Daily Living were also assessed. Behavioural and psychiatric disturbances were evaluated by the Neuropsychiatry Inventory ${ }^{16}$ and Frontal Behavioural Inventory. ${ }^{17}$ Apraxia was assessed by the De Renzi test ${ }^{14}$ and both arms were examined separately.
Assessment of global cognitive functions was carried out according to a standardised battery, blind to clinical diagnosis (as reported in table 1 ).

\section{CSF analyses}

Lumbar puncture was performed on an empty stomach according to a standardised protocol, in the outpatient clinic, from 09:30 to 10:30, after informed written consent had been obtained. CSF was collected in sterile polypropylene tubes and gently mixed to avoid gradient effects. Routine chemical measures were determined. The remaining CSF was centrifuged for $3 \mathrm{~min}$ at $3000 \mathrm{rpm}$, and aliquots were stored at $-80^{\circ} \mathrm{C}$ or in liquid nitrogen for subsequent total tau, phospho-tau and and abeta dosages. CSF concentrations were measured in duplicate by an ELISA test (Innotest hTau antigen kit and Innotest PHOSHO-TAU 181P; Innogenetics, Ghent, Belgium). Interassay variability was less than 7\%. According to our laboratory standards, the cut-off value for total tau is $<400 \mathrm{pg} / \mathrm{ml}$ and for abeta $>400 \mathrm{pg} / \mathrm{ml}$. The AD pattern is defined by high total tau levels and low abeta42 levels in CSF and, as previously reported by other authors, with a CSF tau/abeta42 ratio $>1 .^{18}$

\section{${ }^{99 m}$ Tc-ECD SPECT acquisition and imaging processing}

For brain functional data comparisons, a group of 14 age matched healthy subjects were recruited among patients' spouses or relatives and were included as normal controls. They were interviewed, assessed for neurological or cognitive dysfunction, evaluated for diseases that were exclusion criteria for the patient group, and underwent a brain single photon emission computed tomography (SPECT) study.

Both patients and controls were administered an intravenous injection of $1110 \mathrm{MBq}{ }^{99 \mathrm{~m}} \mathrm{Tc}-\mathrm{ECD}$ (ethylcysteinate dimer, Neurolite; Bristol-Myers Squibb Pharma, New York, USA) under resting conditions, lying supine in a quiet, dimly lit room. All subjects were imaged using a dual head rotating $\gamma$ camera (VG Millenium GE) fitted with a low energy, high resolution collimator, $30 \mathrm{~min}$ after intravenous injection of ${ }^{99 \mathrm{~m}} \mathrm{Tc}-\mathrm{ECD}$.

A $128 \times 128$ pixel matrix was used for image acquisition with 120 views over a $360^{\circ}$ orbit (in $3^{\circ}$ step) with a pixel size and slice

Table 1 Neuropsychological, behavioural and motor assessment according to CSF pattern

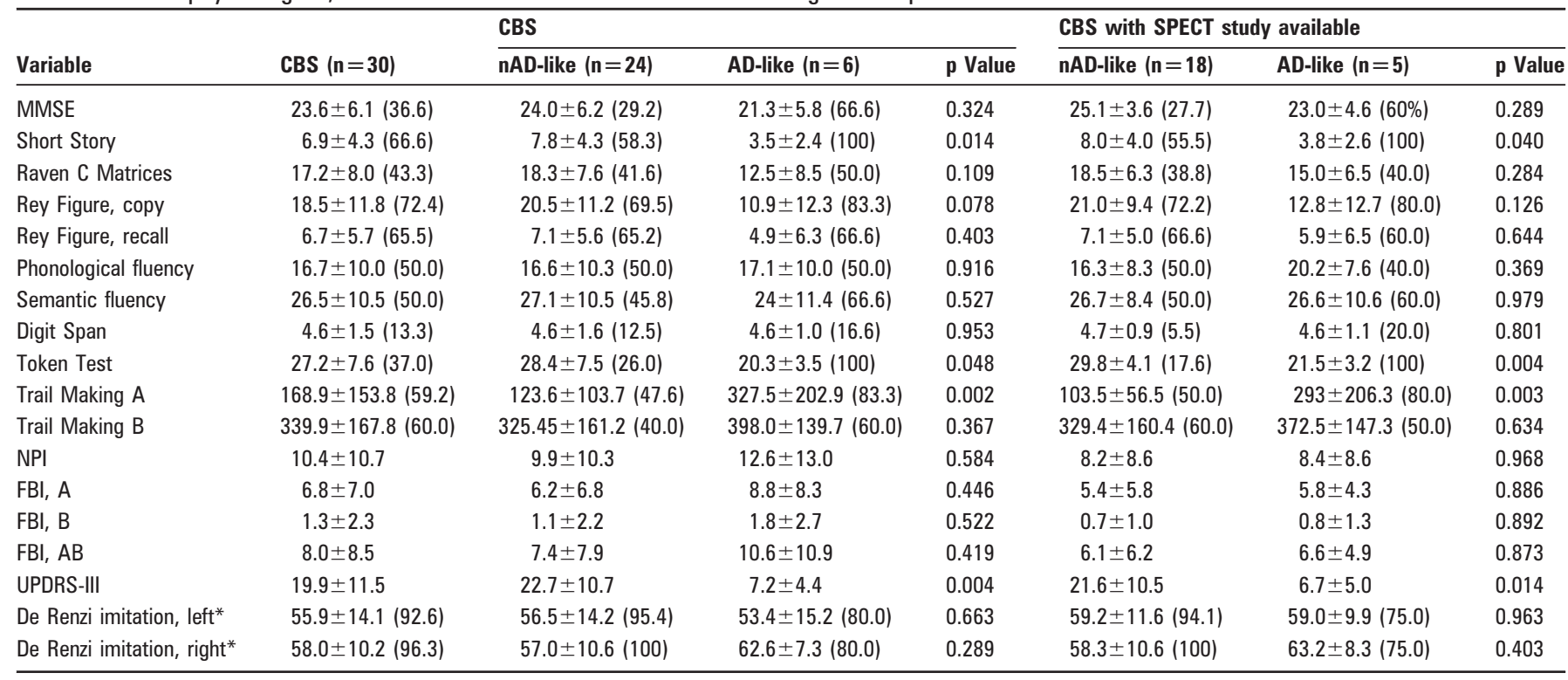

Percentages of patients with pathological scores, according to Italian normative data, are indicated in parentheses.

*De Renzi cut-off value of $<62$ is used.

AD, Alzheimer's disease; CBS, corticobasal syndrome; FBI, Frontal Behavioural Inventory; MMSE, Mini-Mental State Examination; nAD-like, non-Alzheimer's disease-like; NPI, Neuropsychiatry Inventory; SPECT, single photon emission computed tomography; UPDRS-III, Unified Parkinson's Disease Rating Scale. 
Downloaded from jnnp.bmj.com on October 22, 2012 - Published by group.bmj.com

Table 2 Demographic and clinical characteristics according to CSF pattern

\begin{tabular}{|c|c|c|c|c|c|c|c|}
\hline \multirow[b]{2}{*}{ Variables } & \multirow[b]{2}{*}{ CBS $(n=30)$} & \multicolumn{3}{|l|}{ CBS } & \multicolumn{3}{|c|}{ CBS with SPECT study available } \\
\hline & & nAD-like $(n=24)$ & AD-like $(n=6)$ & $\overline{p \text { V Value }}$ & nAD-like $(n=18)$ & AD-like $(n=5)$ & p Value \\
\hline Age at evaluation (years) & $63.5 \pm 8.9$ & $63.6 \pm 9.8$ & $63.0 \pm 4.0$ & 0.882 & $62.2 \pm 10.4$ & $63.6 \pm 4.2$ & 0.779 \\
\hline Age at onset (years) & $61.0 \pm 9.3$ & $60.9 \pm 10.3$ & $61.6 \pm 4.3$ & 0.865 & $60.6 \pm 11.0$ & $62.2 \pm 4.6$ & 0.768 \\
\hline Education (years) & $6.6 \pm 2.9$ & $6.95 \pm 3.2$ & $5.3 \pm 0.8$ & 0.238 & $7.2 \pm 3.2$ & $5.4 \pm 0.9$ & 0.235 \\
\hline Family history (\%) & 13.3 & 12.5 & 16.7 & 0.788 & 5.6 & 20.0 & 0.311 \\
\hline \multicolumn{8}{|l|}{ CSF markers } \\
\hline CSF abeta $(\mathrm{pg} / \mathrm{ml})$ & $748.2 \pm 431.5$ & $865.2 \pm 402.5$ & $280.2 \pm 77.3$ & 0.002 & $889.9 \pm 412.5$ & $266.1 \pm 77.2$ & 0.003 \\
\hline CST tau (pg/ml) & $409.1 \pm 285.3$ & $337.7 \pm 242.5$ & $694.7 \pm 281.7$ & 0.004 & $308.1 \pm 223.1$ & $726.5 \pm 302.7$ & 0.002 \\
\hline CSF tau/abeta ratio & $0.86 \pm 1.03$ & $0.43 \pm 0.29$ & $2.60 \pm 1.20$ & $<0.001$ & $0.44 \pm 0.46$ & $2.83 \pm 1.10$ & $<0.001$ \\
\hline
\end{tabular}

$\mathrm{AD}$, Alzheimer's disease; CBS, corticobasal syndrome; nAD-like, non-Alzheimer's disease-like; SPECT, single photon emission computed tomography.

thickness of $1 \mathrm{~mm}$, in $27 \mathrm{~min}$ or more if total counts were lower than $5 \times 10^{6}$. Image reconstruction was performed by a ramp filtered back projection and three-dimensionally smoothed with a Metz filter (order 3, enhancement 1.24, FWHM $6.7 \mathrm{~mm}$, cut-off 0.61 cycles/cm). The reconstructed images were corrected for $\gamma$ ray attenuation using the Chang method (attenuation coefficient $0.11 / \mathrm{cm}$ ).

Statistical Parametric Mapping (SPM2, Welcome Department of Cognitive Neurology, University College, London, UK) and Matlab 6.1 (Mathworks Inc, Sherborn, Massachusetts, USA) were used for image preprocessing. Images were spatially normalised to a reference stereotactic template (Montreal Neurological Institute) and smoothed by a Gaussian kernel of $8 \times 8 \times 8 \mathrm{~mm}$ FWHM. Global differences in the distribution and the effect of age and gender on the tracer's uptake were covariated out for all voxels. Comparisons across the different groups were made using t statistics with appropriate linear contrasts. We considered any cluster above a statistical threshold set at $\mathrm{p}<0.001$.

\section{Statistical analysis}

Demographic and clinical characteristics between groups were compared by Pearson's $\chi^{2}$ test or the Mann-Whitney test, as appropriate. Statistical significance was assumed at $p<0.05$.

\section{RESULTS}

\section{Subject descriptions}

Mean age at onset was $61.0 \pm 9.3$ and in $60 \%$ of cases the disease began on the left side (see table 2 for demographic characteristics).

In the overall group, mean CSF abeta levels were within the normal range whereas CSF tau levels were slightly increased. As reported in the method section and in figure 1 , according to the

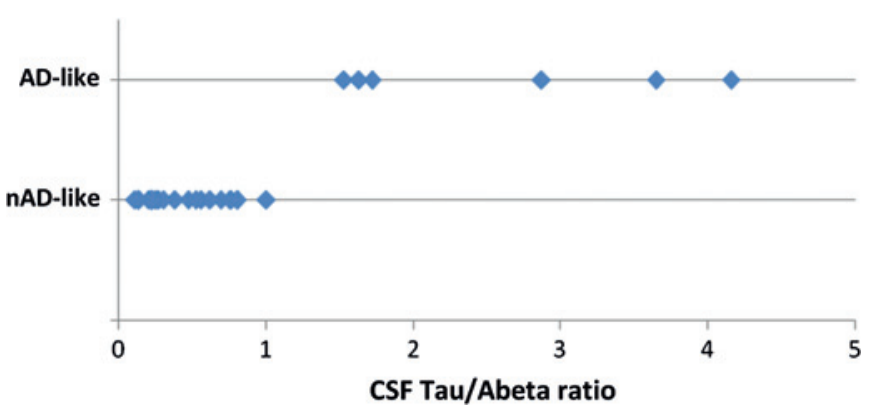

Figure 1 CSF tau/abeta ratio in Alzheimer's disease (AD)-like and nonAD-like groups.
CSF pattern, CBS patients were subdivided into AD-like ( $\mathrm{n}=6$, $20 \%$ ) and nAD-like ( $n=24,80 \%)$ groups. CSF tau and abeta levels as well as CSF tau/abeta ratio are reported in table 2.

\section{Neuropsychological, behavioural and motor assessment}

The neuropsychological, behavioural and motor assessment of CBS patients is presented in table 1. Mean Mini-Mental State Examination score was within the normal range. Behavioural disturbances were present in some CBS patients and the most common disorder was apathy.

$\mathrm{AD}$-like and nAD-like CBS did not differ in terms of global cognitive impairment but the former performed significantly worse in tests assessing episodic memory (Short Story), language comprehension (Token Test) and psychomotor speed (Trail Making A). The performances tapping the other cognitive domains, including praxis (De Renzi test), were comparable, as well as the degree of behavioural disturbances as measured by the Frontal Behavioural Inventory. Conversely, nAD-like CBS showed a more severe extrapyramidal syndrome, as measured by UPDRS-III, compared with AD-like CBS.

\section{Imaging analysis}

Twenty-three out of $30 \mathrm{CBS}$ patients (five $\mathrm{AD}$-like and 18 nAD-like) underwent a SPECT scan (see table 1 for neuropsychological assessment), and hypoperfusion patterns in AD-like versus nAD-like cases were evaluated. Overall, CBS patients showed significantly greater hypoperfusion in the superior $(-48,-46,2, \mathrm{~T}=3.29)$ and middle temporal $(-38,-62$, $18, \mathrm{~T}=2.87)$ gyri and in the inferior parietal lobule $(-50,-28$, $28, T=3.25)$ compared with age matched controls $(p<0.001)$. Accordingly, when the two subgroups of CBS patients (AD-like and $\mathrm{nAD}$-like) were compared with controls, the same pattern of hypoperfusion was observed (AD-like versus controls: -50 , $-44,2, \mathrm{~T}=4.72 ; 54,-32,28, \mathrm{~T}=3.35$; and $\mathrm{nAD}$-like versus controls: $-40,-56,14, \mathrm{~T}=2.94)$.

As reported in table 3 and figure 2, in AD-like CBS, greater hypoperfusion of the hippocampus and parahyppocampal gyrus, posterior cingulate and precuneus, bilaterally, was demonstrated $(\mathrm{AD}<\mathrm{nAD}, \mathrm{p}<0.001)$ than in $\mathrm{nAD}$-like CBS. Conversely, no voxels above the pre-established threshold were identified when $\mathrm{nAD}$-like patients were compared with $\mathrm{AD}$-like patients $(\mathrm{nAD}<\mathrm{AD})$.

\section{DISCUSSION}

In the present work, we reported that patients with classic CBS have two distinct profiles of CSF tau/abeta ratio, $20 \%$ presenting with an AD-like pattern. No differences in demographic 
Table 3 Location of the peaks of reduction of regional cerebral blood flow in corticobasal syndrome with CSF AD-like pattern compared with nAD-like pattern

\begin{tabular}{|c|c|c|c|c|c|c|}
\hline \multirow[b]{2}{*}{ Brain region } & \multicolumn{3}{|c|}{ Peak coordinates (mm) } & \multirow[b]{2}{*}{$\mathbf{T}$} & \multirow[b]{2}{*}{ p Value } & \multirow[b]{2}{*}{ Cluster size } \\
\hline & $\mathbf{x}$ & y & $\mathbf{z}$ & & & \\
\hline \multicolumn{7}{|c|}{ CBS-AD versus CBS-nAD $(A D<n A D)$} \\
\hline Precuneus (R) & 18 & -52 & 32 & 5.77 & $<0.001$ & 621 \\
\hline Posterior cingulate (R) & 16 & -42 & 10 & 4.23 & $<0.001$ & - \\
\hline Hippocampus (R) & 24 & -16 & -18 & 5.41 & $<0.001$ & 937 \\
\hline Parahippocampal gyrus (R) & 26 & -2 & -12 & 3.77 & $<0.001$ & - \\
\hline Precuneus (L) & -18 & -44 & 46 & 4.79 & $<0.001$ & 1390 \\
\hline Posterior cingulate (L) & -20 & -70 & 20 & 4.72 & $<0.001$ & 2175 \\
\hline Hippocampus (L) & -30 & -40 & -10 & 4.81 & $<0.001$ & - \\
\hline Parahippocampal gyrus (L) & -38 & -42 & -8 & 4.91 & $<0.001$ & - \\
\hline \multicolumn{7}{|c|}{ CBS-nAD versus $C B S-A D(n A D<A D)$} \\
\hline None & - & - & - & - & - & - \\
\hline
\end{tabular}

Corticobasal syndrome; $x, y$ and $z$ values localise the areas of regional cerebral blood flow reduction according to the Montreal Neurological Institute stereotactic coordinates.

$A D$, Alzheimer's disease; CBS, corticobasal syndrome; L, left; $n A D$, non-Alzheimer's disease-like; $R$, right.

characteristics were detected between groups, and apraxia scores were comparable. Compared with $\mathrm{nAD}$-like CBS, AD-like patients reported earlier memory impairment as well as worse psychomotor speed and language dysfunctions. Conversely, $n A D$-like patients had a more severe akinetic extrapyramidal syndrome. Finally, in AD-like CBS patients, CSF and neuropsychological profiles were corroborated by greater hypoperfusion in those areas typically affected by $\mathrm{AD}$ - namely, the hippocampus, parahyppocampal region, precuneus and posterior cingulate, bilaterally.

A few studies on CSF markers in CBS are already available, reporting conflicting results. In two studies, CSF tau protein dosage was significantly higher in CBS than in healthy controls ${ }^{1920}$ whereas three other studies showed no significant differences between CBS and patients affected by neurodegenerative diseases with distinct tau related pathology. ${ }^{21-23}$

However, despite the conflicting results and lack of comparison groups, all of the above studies reported high variability in CSF markers, arguing for different associated neuropathological features.

In clinically defined CBS, CSF findings are still unpredictable but it is widely demonstrated that $\mathrm{AD}$ is associated with a specific pattern of CSF abnormalities-that is, high tau and low abeta levels compared with other neurodegenerative
Figure 2 Reduction of regional cerebral blood flow in corticobasal syndrome (CBS) with CSF Alzheimer's disease $(A D)$-like pattern compared with CSF non-AD-like pattern. Greater hypoperfusion in the hyppocampi (top row) and posterior cingulate (bottom row) in AD-like versus non-AD-like CBS patients is reported. $L$, left; $R$, right.

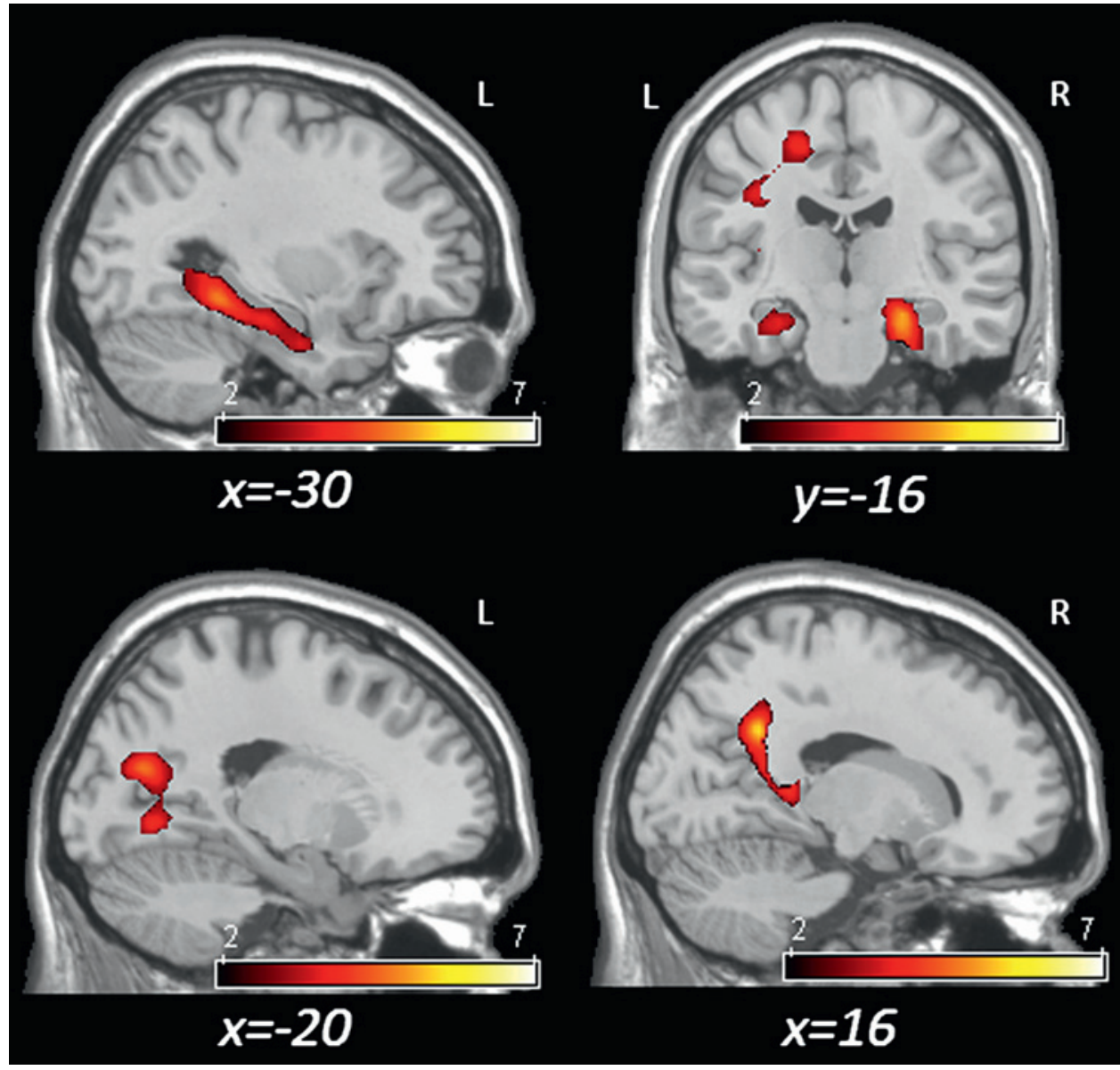


disorders. ${ }^{11}$ To date, the CSF tau/abeta marker has been strongly suggested as a supportive criterion for early and preclinical $\mathrm{AD}$ diagnosis. $^{13}$

Herein, we have tested the hypothesis that different CSF patterns - that is, $\mathrm{AD}$-like and $\mathrm{nAD}$-like-might define distinct CBS phenotypes. In fact, although diagnostic criteria for CBS arose from clinical cases with pathologically confirmed $\mathrm{CBD},{ }^{24}$ it has been shown that $\mathrm{AD}$ can also be a common cause of CBS. ${ }^{4} 8102526$ This pathological heterogeneity likely reflects the range of neurodegenerative disorders which can present with asymmetric frontal and/or parietal lobar dysfunction, ${ }^{10}$ but clinical or neuropsychological CBS features useful to distinguish $\mathrm{AD}$ cases are not yet clear. One study evaluated 12 patients with CBS who had been followed prospectively; six had $\mathrm{AD}$ and six had classic CBD neuropathology. After review of the presenting clinical features, the authors reported that the initial episodic memory complaints and poor performance on the combined orientation-memory subtest of the Addenbrooke's Cognitive Examination reliably predicted $\mathrm{AD}$ pathology. ${ }^{9}$

We also demonstrated that patients with a CSF AD-like pattern had greater hypoperfusion in those brain areas typically affected by classic $\mathrm{AD}$-namely, the hippocampus, parahyppocampal region, precuneus and posterior cingulate. These brain areas have been found to be affected even from the early stages and represent the preclinical neuroimaging hallmarks of those subjects who will develop a symptomatic dementia of the $\mathrm{AD}$ type. ${ }^{27}$ Up until now, no other neurodegenerative disorder has been characterised by these neuroimaging features, claiming that the presence of hippocampal and posterior cingulate hypoperfusion is suggestive of $\mathrm{AD} .^{27}$

We may alternatively hypothesise that CSF formula, along with neuroimaging findings suggestive for $\mathrm{AD}$ pathology, may herald in these CBS patients the onset of a dementia that can develop in their later life.

We acknowledge that this work has some limits. Firstly, the neuropathological confirmation and the combined use of amyloid $\beta$ tracers would be mandatory to definitively clarify the present findings. Secondly, a more careful evaluation of memory deficits might be of help for further differentiating $\mathrm{AD}$-like and nAD-like subgroups. Thirdly, the neuroimaging data analysis herein was uncorrected for multiple comparisons, thus limiting the conclusions that can be derived. These need further validation in larger and independently assessed cohorts.

Moreover, it is interesting to observe that total tau levels in CSF were normal for the majority of our CBS cases. We can therefore argue that the tauopathy found at neuronal and glial cytoplasmic inclusions at autopsy is not detectable by this ELISA, but other technologies should be developed to measure and disentangle the molecular mechanisms of tau deposition in CBS.

However, taken together, these data suggest that the CSF tau/ abeta ratio might be a potential avenue for detecting $\mathrm{AD}$ cases presenting with CBS. In the near future, CSF data alone, or in combination with other neuropsychological or neuroimaging markers, might improve the accuracy of an $\mathrm{AD}$-like diagnosis and might be included in research criteria to identify a distinct population. If confirmed by future studies, patients with an AD-like pattern should undergo a different therapeutic approach, and current pharmacological treatment for $\mathrm{AD}$ should be considered.
Acknowledgements The authors are indebted to Dr Andrew Harwood for editing the manuscript.

Competing interests None.

Ethics approval This study was conducted with the approval of the Brescia Hospital Ethics Committee.

Provenance and peer review Not commissioned; externally peer reviewed.

\section{REFERENCES}

1. Rebeiz JJ, Kolodny EH, Richardson EP Jr. Corticodentatonigral degeneration with neuronal achromasia. Arch Neurol 1968;18:20-33.

2. Lang AE. Corticobasal degeneration: selected developments. Mov Disord 2003;18 S51-6.

3. Litvan I, Bhatia KP, Burn DJ, et al. Movement Disorders Society Scientific Issues Committee report: SIC Task Force appraisal of clinical diagnostic criteria for Parkinsonian disorders. Mov Disord 2003:18:467-86.

4. Boeve BF, Maraganore DM, Parisi JE, et al. Pathologic heterogeneity in clinically diagnosed corticobasal degeneration. Neurology 1999;53:795-800.

5. Ball JA, Lantos PL, Jackson $\mathbf{M}$, et al. Alien hand sign in association with Alzheimer's histopathology. J Neurol Neurosurg Psychiatry 1993;56:1020-3.

6. Green RC, Goldstein FC, Mirra SS, et al. Slowly progressive apraxia in Alzheimer's disease. J Neurol Neurosurg Psychiatry 1995;59:312-15.

7. Doran M, du Plessis DG, Enevoldson TP, et al. Pathological heterogeneity of clinically diagnosed corticobasal degeneration. J Neurol Sci 2003;216:127-34.

8. Chand P, Grafman J, Dickson D, et al. Alzheimer's disease presenting as corticobasal syndrome. Mov Disord 2006;21:2018-22.

9. Shelley BP, Hodges JR, Kipps CM, et al. Is the pathology of corticobasal syndrome predictable in life? Mov Disord 2009;24:1593-9.

10. Hu WT, Rippon GW, Boeve BF, et al. Alzheimer's disease and corticobasal degeneration presenting as corticobasal syndrome. Mov Disord 2009;24:1375-9.

11. Mattsson N, Zetterberg $\mathrm{H}$, Hansson 0, et al. CSF biomarkers and incipient Alzheimer disease in patients with mild cognitive impairment. JAMA 2009;302:385-93.

12. Visser PJ, Verhey F, Knol DL, et al. Prevalence and prognostic value of CSF markers of Alzheimer's disease pathology in patients with subjective cognitive impairment or mild cognitive impairment in the DESCRIPA study: a prospective cohort study. Lancet Neurol 2009;8:619-27.

13. Dubois B, Feldman $\mathrm{HH}$, Jacova $\mathrm{C}$, et al. Research criteria for the diagnosis of Alzheimer's disease: revising the NINCDS-ADRDA criteria. Lancet Neurol 2007;6:734-46.

14. De Renzi E, Motti F, Nichelli P. Imitating gestures. A quantitative approach to ideomotor apraxia. Arch Neurol 1980;37:6-10.

15. Fahn S, Elton RL. Members of the UPDRS development committee. Unified Parkinson's Disease rating Scale. Florham Park, NJ: MacMillan Healthcare Information, 1987:153-304.

16. Cummings JL, Mega M, Gray K, et al. The Neuropsychiatric Inventory: comprehensive assessment of psychopathology in dementia. Neurology 1994;44:2308-14.

17. Kertesz A, Nadkarni N, Davidson W, et al. The Frontal Behavioral Inventory in the differential diagnosis of frontotemporal dementia. J Int Neuropsychol Soc 2000;6:460-8.

18. Bian H, Van Swieten JC, Leight S, et al. CSF biomarkers in frontotemporal lobar degeneration with known pathology. Neurology 2008;70:1827-35.

19. Urakami K, Wada K, Arai $\mathrm{H}$, et al. Diagnostic significance of tau protein in cerebrospinal fluid from patients with corticobasal degeneration or progressive supranuclear palsy. J Neurol Sci 2001;183:95-8.

20. Paraskevas GP, Kapaki E, Liappas I, et al. The diagnostic value of cerebrospinal fluid tau protein in dementing and nondementing neuropsychiatric disorders. J Geriatr Psychiatry Neurol 2005;18:163-73.

21. Arai H, Morikawa $Y$, Higuchi $M$, et al. Cerebrospinal fluid tau levels in neurodegenerative diseases with distinct tau-related pathology. Biochem Biophys Res Commun 1997;236:262-4.

22. Borroni B, Malinverno M, Gardoni F, et al. Tau forms in CSF as a reliable biomarker for progressive supranuclear palsy. Neurology 2008;71:1796-803.

23. Noguchi M, Yoshita M, Matsumoto $Y$, et al. Decreased beta-amyloid peptide42 in cerebrospinal fluid of patients with progressive supranuclear palsy and corticobasal degeneration. J Neurol Sci 2005;237:61-5.

24. Litvan I, Agid Y, Goetz C, et al. Accuracy of the clinical diagnosis of corticobasal degeneration: a clinicopathologic study. Neurology 1997;48:119-25.

25. Alladi S, Xuereb J, Bak T, et al. Focal cortical presentations of Alzheimer's disease. Brain 2007; 130:2636-45

26. Okazaki K, Fu YJ, Nishihira Y, et al. Alzheimer's disease: report of two autopsy cases with a clinical diagnosis of corticobasal degeneration. Neuropathology 2010;30:140-8.

27. Anchisi D, Borroni B, Franceschi M, et al. Heterogeneity of brain glucose metabolism in mild cognitive impairment and clinical progression to Alzheimer disease. Arch Neurol 2005;62:1728-33. 


\section{JNNP}

\section{CSF Alzheimer's disease-like pattern in corticobasal syndrome: evidence for a distinct disorder}

Barbara Borroni, Enrico Premi, Chiara Agosti, et al.

J Neurol Neurosurg Psychiatry 2011 82: 834-838 originally published online February 22, 2011

doi: 10.1136/jnnp.2010.221853

Updated information and services can be found at:

http://jnnp.bmj.com/content/82/8/834.full.html

These include:

References This article cites 26 articles, 9 of which can be accessed free at: http:/jnnnp.bmj.com/content/82/8/834.full.html\#ref-list-1

Email alerting Receive free email alerts when new articles cite this article. Sign up in service the box at the top right corner of the online article.

$\begin{array}{cc}\text { Topic } & \text { Articles on similar topics can be found in the following collections } \\ \text { Collections } & \text { Neuropathology (147 articles) } \\ & \text { Stroke (1105 articles) } \\ \text { Memory disorders (psychiatry) (999 articles) } \\ \text { Dementia (746 articles) } \\ \text { Drugs: CNS (not psychiatric) (1347 articles) } \\ \text { Psychiatry of old age (235 articles) } \\ \text { Radiology (1372 articles) } \\ \text { Radiology (diagnostics) (1041 articles) }\end{array}$

Notes

To request permissions go to:

http://group.bmj.com/group/rights-licensing/permissions

To order reprints go to:

http://journals.bmj.com/cgi/reprintform

To subscribe to BMJ go to:

http://group.bmj.com/subscribe/ 